\title{
Anti-angiogenic Therapy in Patients with Advanced Gastric and Gastroesophageal Junction Cancer: A Systematic Review
}

\section{Li-Tzong Chen, $\mathrm{MD}^{1}$ \\ Do-Youn Oh, MD² \\ Min-Hee Ryu, MD ${ }^{3}$ \\ Kun-Huei Yeh, $\mathrm{MD}^{4}$ \\ Winnie Yeo, MD \\ Roberto Carlesi, MD 6 \\ Rebecca Cheng, $\mathrm{MD}^{7}$ \\ Jongseok Kim, MD \\ Mauro Orlando, $\mathrm{MD}^{9}$ \\ Yoon-Koo Kang, MD ${ }^{3}$}

\author{
${ }^{1}$ National Institute of Cancer Research, \\ National Health Research Institutes and \\ National Cheng Kung University Hospital, \\ National Cheng Kung University, Tainan, \\ Taiwan, ${ }^{2}$ Division of Medical Oncology, \\ Department of Internal Medicine, \\ Seoul National University Hospital, Cancer \\ Research Institute, Seoul National University \\ College of Medicine, Seoul, ${ }^{3}$ Department of \\ Oncology, Asan Medical Center, \\ University of Ulsan College of Medicine, \\ Seoul, Korea, ${ }^{4}$ Department of Oncology, \\ National Taiwan University Hospital, Taipei, \\ Taiwan, ${ }^{5}$ Department of Clinical Oncology, \\ The Chinese University of Hong Kong, \\ Hong Kong, China, ${ }^{6}$ Eli Lilly and Company, \\ Sesto Fiorentino, Florence, Italy, \\ ${ }^{7}$ Eli Lilly and Company, Taipei, Taiwan, \\ ${ }^{8}$ Eli Lilly and Company, Indianapolis, \\ IN, USA, ${ }^{9}$ Eli Lilly and Company, \\ Buenos Aires, Argentina
}

Despite advancements in therapy for advanced gastric and gastroesophageal junction cancers, their prognosis remains dismal. Tumor angiogenesis plays a key role in cancer growth and metastasis, and recent studies indicate that pharmacologic blockade of angiogenesis is a promising approach to therapy. In this systematic review, we summarize current literature on the clinical benefit of anti-angiogenic agents in advanced gastric cancer. We conducted a systematic search of PubMed and conference proceedings including the American Society of Clinical Oncology, the European Society for Medical Oncology, and the European Cancer Congress. Included studies aimed to prospectively evaluate the efficacy and safety of anti-angiogenic agents in advanced gastric or gastroesophageal junction cancer. Each trial investigated at least one of the following endpoints: overall survival, progression-free survival/time to progression, and/or objective response rate. Our search yielded 139 publications. Forty-two met the predefined inclusion criteria. Included studies reported outcomes with apatinib, axitinib, bevacizumab, orantinib, pazopanib, ramucirumab, regorafenib, sorafenib, sunitinib, telatinib, and vandetanib. Second-line therapy with ramucirumab and third-line therapy with apatinib are the only anti-angiogenic agents so far shown to significantly improve survival of patients with advanced gastric cancer. Overall, agents that specifically target the vascular endothelial growth factor ligand or receptor have better safety profile compared to multi-target tyrosine kinase inhibitors.

\author{
Key words \\ Angiogenesis inhibitors, Esophagogastric junction, \\ Stomach neoplasms, Vascular endothelial growth factors
}

\author{
Correspondence: Yoon-Koo Kang, MD \\ Department of Oncology, Asan Medical Center, \\ University of Ulsan College of Medicine, \\ 88 Olympic-ro 43-gil, Songpa-gu, \\ Seoul 05505, Korea \\ Tel: 82-2-3010-5001 \\ Fax: 82-2-3010-8772 \\ E-mail: ykkang@amc.seoul.kr \\ Received April 26, 2016 \\ Accepted December 20, 2016 \\ Published Online January 3, 2017
}




\section{Introduction}

Gastric cancer is the fifth most common malignancy and the third leading cause of cancer mortality worldwide [1]. Curative intent surgical resection is the preferred approach to treat localized gastric cancer. Despite radical resection and perioperative or adjuvant treatment, recurrence rates remain high in gastric cancer patients, with a consequently poor prognosis [2]. Platinum plus fluoropyrimidine-based combinations are established worldwide as first-line drug regimens [3]. In randomized trials, selected second-line chemotherapy significantly improved overall survival (OS) compared with best supportive care; however, median survival was less than six months with second-line chemotherapy [4].

Vascular endothelial growth factor (VEGF) and VEGF receptor-2 (VEGFR-2)-mediated signaling and angiogenesis contribute to the pathogenesis of gastric cancer. In patients with gastric cancer, circulating and tumor concentrations of VEGF are associated with increased tumor aggressiveness and reduced survival [5]. In preclinical studies, inhibition of VEGF or VEGFR-2, or their downstream signaling pathways, has been shown to reduce tumor growth [6]. Anti-angiogenic agents have been approved for a range of cancer types, and more recently in advanced gastric or gastroesophageal junction cancer.

We systematically reviewed the literature to evaluate the efficacy and safety of anti-angiogenic agents in advanced gastric or gastroesophageal junction cancer, with a focus on the safety profiles of agents with different mechanisms of action, quality of life (QoL), and biomarkers of response.

\section{Mechanisms of Action of Anti-angiogenic Agents}

VEGF-A is a key regulator of angiogenesis [7]. The VEGFVEGFR signaling axis is composed of multiple ligands and receptors with overlapping and distinct ligand-receptor binding specificities, cell-type expression, and function [8]. Activation of the VEGFR-2 pathway triggers a network of signaling processes that stimulate endothelial cell growth, migration, and survival from preexisting vasculature [7]. In addition, VEGF-A mediates vessel permeability and has been associated with malignant effusions and the mobilization of endothelial progenitor cells from the bone marrow to distant sites of neovascularization [7].

Studies with various anti-angiogenic agents have shown that these agents can inhibit angiogenesis and tumor growth in preclinical models. Clinical studies show anti-angiogenic agents, either alone or in combination with chemotherapy, can significantly improve survival and response rates in a range of cancer types. The most common approaches to inhibiting the VEGF-VEGFR signaling axis include VEGF ligand-targeted therapy, inhibition of VEGFR tyrosine kinases and their downstream targets, and VEGFR-2-targeted therapy (S1 Fig.). Other approaches include blockade of angiopoietin1-Tie2 signaling, which is essential for developmental vascular formation [9], destabilizing of tumor vasculature and its supporting structures with vascular disrupting agents [10], and inhibition of hypoxia-inducible factors [11]; these agents were not in clinical development in gastric cancer at the time of this systematic search and are beyond the scope of this review.

\section{Methods}

\section{Data sources and searches}

This review and its procedures were planned, conducted, and reported according to the Preferred Reporting Items for Systematic Reviews and Meta-Analyses (PRISMA) guidelines [12]. A comprehensive search strategy was developed for PubMed (S2 Table). Congress abstract databases do not have an advanced search function; therefore, pairs of terms were derived from the search strategy for PubMed and used to search the following congress abstract databases: American Society for Clinical Oncology (ASCO) Annual Meeting, ASCO Gastrointestinal Cancers Symposium, European Society for Medical Oncology (ESMO) Congress, ESMO World Congress on Gastrointestinal Cancer, and European Cancer Congress (S3 Table). Additional articles were identified by searches of a range of bibliographic sources, including ClinicalTrials.gov. The search was not restricted by publication date, with the exception of congress abstract databases, which were limited to the past 2 years. The primary search was conducted in July 2015, and a second search conducted in September 2016 to identify articles published after July 2015 when the initial search was performed.

\section{Study selection}

Two authors (R.C. and R.C.) were designated as reviewers and independently screened the title and abstract of each record retrieved to identify potentially relevant articles for full-text review. In cases in which the two reviewers did not agree, a third reviewer arbitrated. Inclusion criteria included meta-analyses; systematic reviews; randomized and nonrandomized clinical trials; retrospective and prospective obser- 


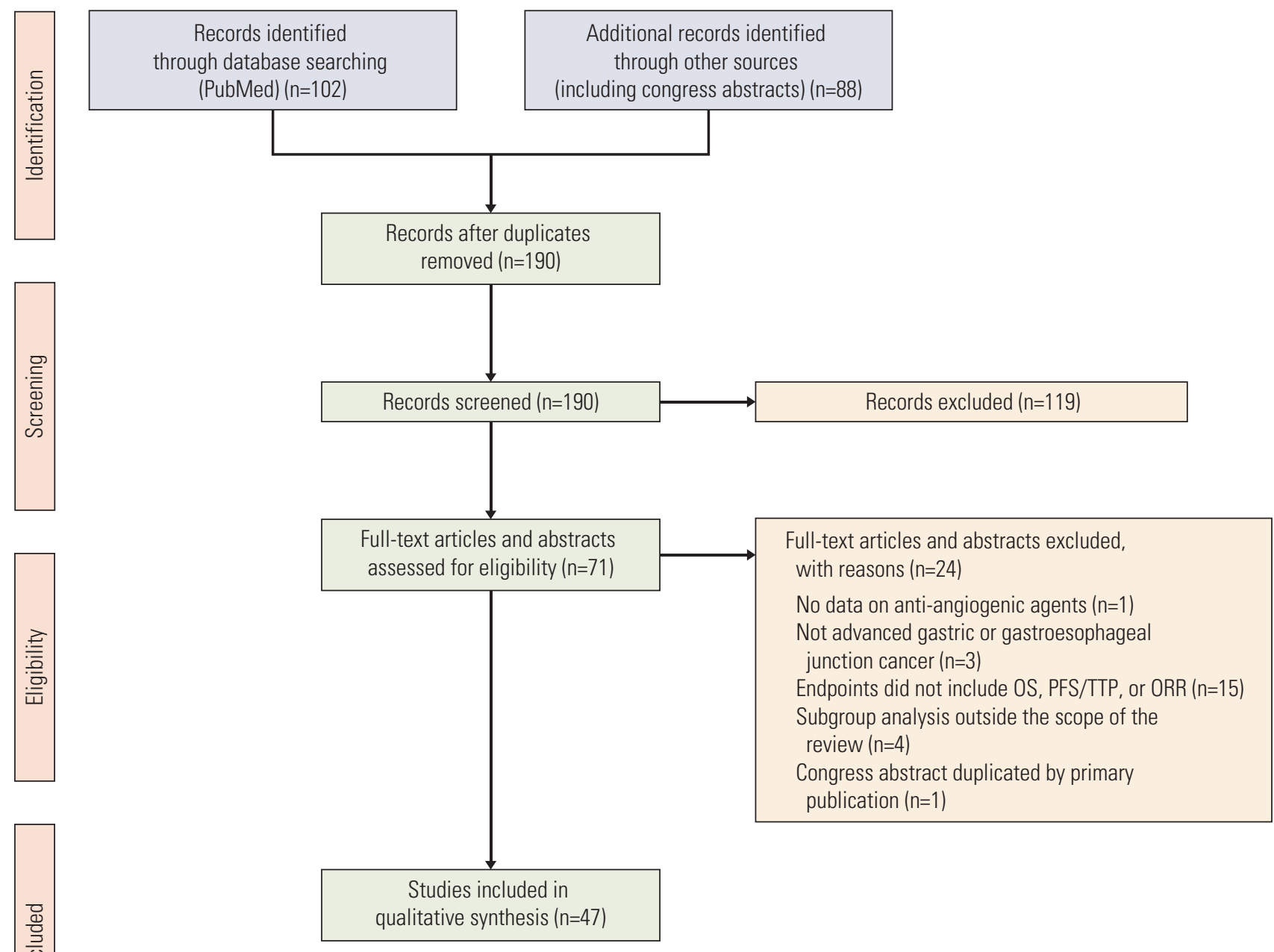

Fig. 1. PRISMA flow diagram. OS, overall survival; PFS, progression-free survival; TTP, time to progression; ORR, objective response rate.

vational studies; phase I, II, and III clinical trials; studies of patients with advanced gastric cancer; and studies on antiangiogenic agents. Each trial investigated at least one of the following endpoints: OS, progression-free survival (PFS)/ time to progression (TTP), and/or objective response rate (ORR); complete response plus partial response (CR+PR). Exclusion criteria included narrative reviews, letters, editorials, commentaries, case studies, case series, non-human studies, studies of patients with early-stage gastric cancer, studies of patients with gastrointestinal stromal tumors, studies on colorectal cancer, and studies not published in English.

\section{Results}

Our search yielded 189 publications, 47 of which met the predefined inclusion criteria. Included studies reported outcomes with apatinib, axitinib, bevacizumab, orantinib, pazopanib, ramucirumab, regorafenib, sorafenib, sunitinib, telatinib, and vandetanib. The PRISMA flow diagram for this systematic review is shown in Fig. 1. 


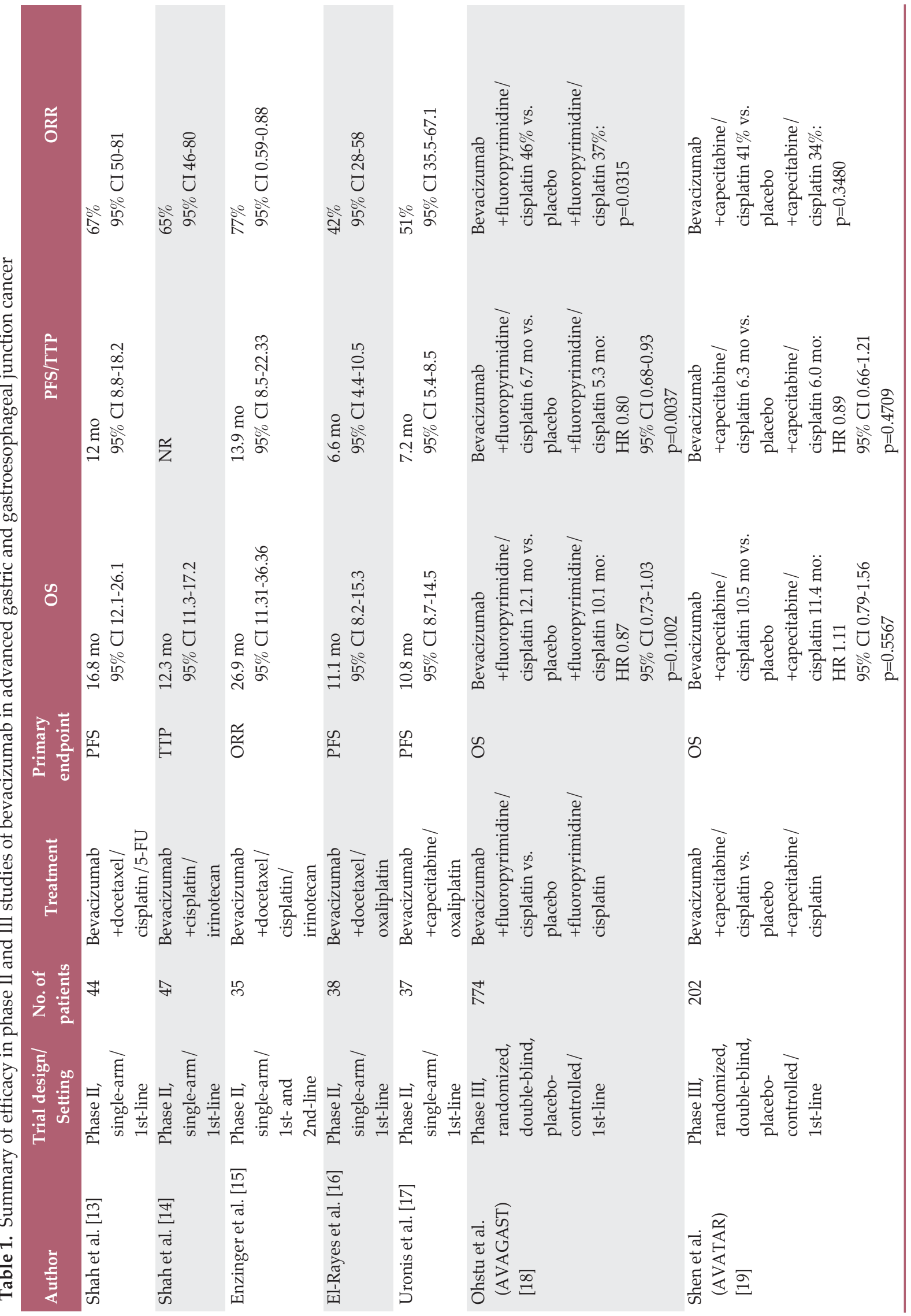




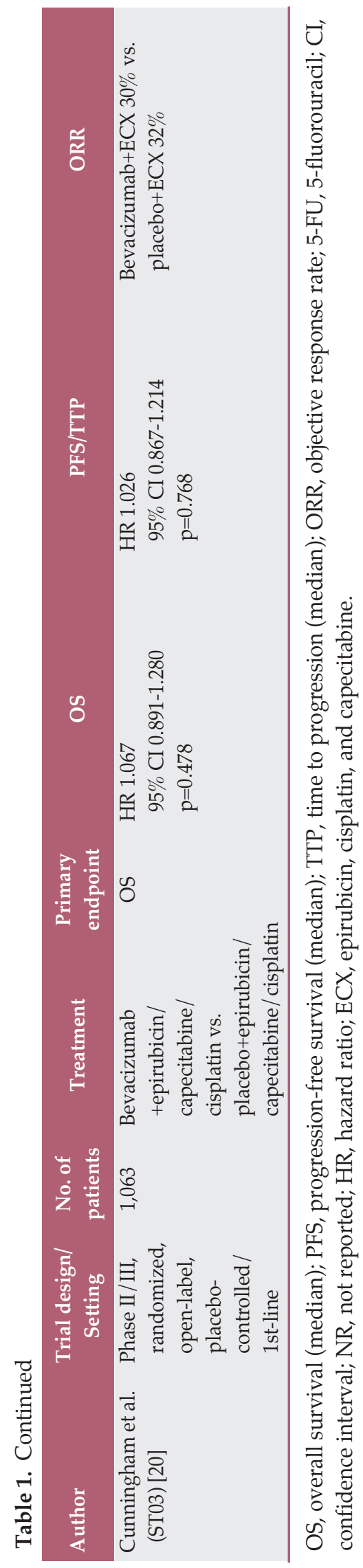

\section{Efficacy and safety of VEGF-A-targeted therapy}

Bevacizumab and ziv-aflibercept are VEGF ligand-targeted therapies. Bevacizumab is a monoclonal antibody targeting VEGF-A, a key mediator of physiologic and pathologic angiogenesis while the recombinant fusion protein zivaflibercept binds both VEGF-A and -B and placental growth factor.

A summary of published phase II and III trials with bevacizumab in gastric cancer is shown in Table 1. Multiple phase II single-arm studies of bevacizumab in combination with first-line chemotherapy in advanced gastric cancer showed that median OS ranged from 10.8 months to 16.8 months [13-17].

A phase III, international, randomized, double-blind, placebo-controlled study (AVAGAST) investigated bevacizumab in combination with fluoropyrimidine-cisplatin in the first-line setting in 774 patients with advanced or metastatic gastric or gastroesophageal junction cancer [18]. Although median PFS and ORR were significantly improved with addition of bevacizumab, this study did not meet the primary endpoint of OS (Table 1). The most common treatment emergent adverse events were similar between the treatment arms (grade 3-5 neutropenia [35\% vs. 37\%], anemia [10\% vs. $14 \%$, and decreased appetite [ $8 \%$ vs. $11 \%]$ ). Adverse events of special interest (AESIs) included grade 3-5 venous thromboembolic events (6\% vs. 9\%), and hypertension $(6 \%$ vs. $<1 \%)$.

Interestingly, in a preplanned exploratory subgroup analysis of the AVAGAST study, there was a difference in the benefit from bevacizumab among the geographic regions of the world. Pan-American patients appeared to have a survival benefit (11.5 months vs. 6.8 months; hazard ratio [HR], 0.63; $95 \%$ confidence interval [CI], 0.43 to 0.94 ) whereas Asian patients appeared to have no benefit (13.9 months vs. 12.1 months; HR, 0.97; 95\% CI, 0.75 to 1.25), and European patients had an intermediate benefit (11.1 months vs. 8.6 months; HR, $0.85 ; 95 \%$ CI, 0.63 to 1.14 ), possibly due to differences in independent prognostic factors and use of subsequent therapies [18].

Based on the geographical differences observed in the AVAGAST study, a study of similar design (AVATAR) was conducted in 202 Chinese patients [19]. Similarly to AVAGAST, AVATAR did not reach its primary endpoint and showed similar median PFS and ORR between the two arms (Table 1). Treatment emergent adverse event (TEAEs) and AESIs were lower for the bevacizumab compared to the control arm (TEAEs, grade 3-5 hemorrhage [4\% vs. $12 \%$ ]; grade $\geq 3$ AESIs, hemorrhage [ $4 \%$ vs. $12 \%$ ] and arterial thromboembolic events [3\% vs. $4 \%]$ ).

A phase II/III, multicenter, randomized study (ST03) investigated perioperative epirubicin, capecitabine, and cis- 


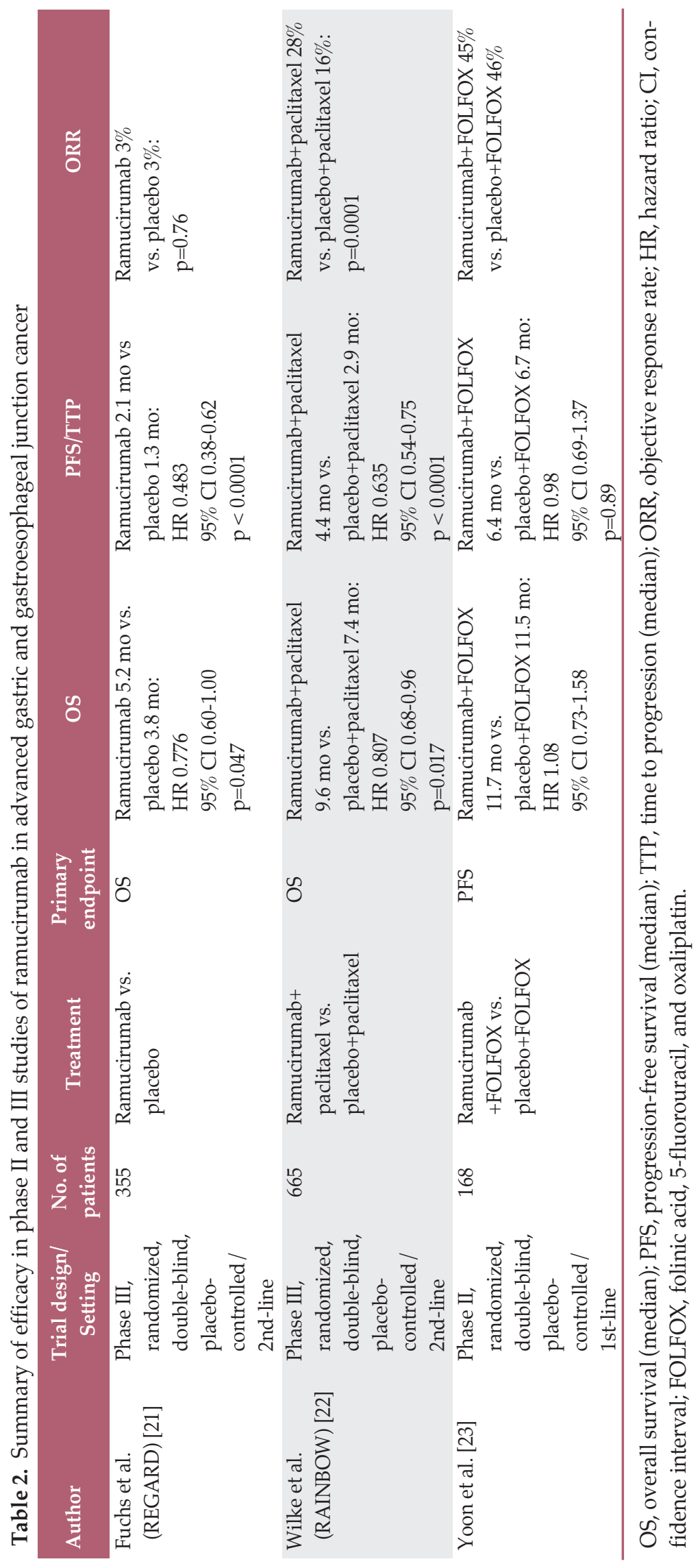


platin with or without bevacizumab in 1,063 patients with localized gastric or gastroesophageal junction cancer [20,24]. Likewise, 3-year survival rates (48\% vs. $49 \%$ ) and diseasefree survival (HR, 1.006; $95 \% \mathrm{CI}, 0.852$ to $1.187 ; \mathrm{p}=0.943$ ) were not significantly different between the treatment arms. The most common grade $\geq 3$ TEAEs included lethargy and handfoot syndrome which were similar, and neutropenia which was slightly higher in the bevacizumab arm (S4 Table).

The first data from the phase II, double-blind, randomized study of ziv-aflibercept for the first-line treatment of 64 patients with metastatic gastric and gastroesophageal junction cancer was disclosed at the ASCO 2016 Gastrointestinal Cancers Symposium [25]. This study showed that ziv-aflibercept did not significantly improve the efficacy of folinic acid, 5-fluorouracil, and oxaliplatin (FOLFOX). The 6-month PFS rate was $60.5 \%$ in the ziv-aflibercept plus FOLFOX arm and $57.1 \%$ in the placebo plus FOLFOX $\operatorname{arm}(\mathrm{p}=0.80)$. Grade $\geq 3$ TEAEs included hypertension ( $47 \%$ vs. $5 \%)$, neutropenia ( $28 \%$ vs. $19 \%$ ), and fatigue (12\% vs. $5 \%$ ) [25].

Based on these published studies, there is currently no established role for targeting VEGF-A for first-line therapy in gastric cancer. There are however several ongoing studies of bevacizumab which are described in S5 Table.

\section{Efficacy and safety of VEGFR-2-targeted therapy}

Ramucirumab is a recombinant human immunoglobulin G1 (IgG1) neutralizing monoclonal antibody specific for VEGFR-2 that prevents ligand binding and receptor-mediated pathway activation in endothelial cells [26]. Ramucirumab is approved as a single agent, or in combination with paclitaxel, for the treatment of patients with advanced or metastatic gastric or gastroesophageal junction cancer with disease progression on or after prior fluoropyrimidineor platinum-containing chemotherapy. Ramucirumab is also approved in the second-line setting in metastatic non-small cell lung cancer and metastatic colorectal cancer. A summary of published phase II and III trials with ramucirumab in gastric cancer is shown in Table 2.

A phase III, international, randomized, double-blind, placebo-controlled study (REGARD) investigated ramucirumab versus placebo in the second-line setting in 355 patients with advanced gastric or gastroesophageal junction cancer [21]. To our knowledge, this study was the first to show that a biological treatment given as a single drug has survival benefits as assessed by OS and PFS in patients with gastric cancer (Table 2). The most common grade $\geq 3$ TEAEs were hypertension, fatigue, abdominal pain, and anemia which all had a similar incidence between treatment arms (S4 Table). Rates of any-grade TEAEs were mostly similar between treatment arms (94\% vs. $88 \%$ ). AESIs included hypertension ( $16 \%$ vs. $8 \%$ ) and bleeding or hemorrhage
( $13 \%$ vs. $11 \%)$.

A subgroup analysis of the REGARD study showed that the survival benefit associated with ramucirumab was similar between Asian patients and those from America, Europe, and Australia, although relatively few Asian patients were enrolled (Caucasian patients, median OS [HR, 0.784; 95\% CI, 0.590 to 1.042]; Asian patients, median OS [HR, 0.636; 95\% CI, 0.306 to 1.321]) [21]. When compared to placebo, ramucirumab conferred similar improvements for median OS and PFS between patients aged $<65$ years and those aged $\geq 65$ years in the REGARD study (median OS [HR, 0.846; 95\% CI, 0.611 to 1.171 ] vs. [HR, $0.722 ; 95 \% \mathrm{CI}, 0.471$ to 1.106 ]; median PFS [HR, 0.450; 95\% CI, 0.327 to 0.620 ] vs. [HR, $0.490 ; 95 \%$ CI, 0.319 to 0.752$]$ ) [27].

A second phase III, international, randomized, doubleblind, placebo-controlled study (RAINBOW) investigated ramucirumab plus paclitaxel versus placebo plus paclitaxel in the second-line setting in 665 patients with advanced gastric or gastroesophageal junction cancer [22]. This study showed that the combination of ramucirumab with paclitaxel significantly improved median OS, median PFS, and ORR (Table 2), and could be regarded as a new standard secondline treatment for patients with advanced gastric cancer. The most common grade $\geq 3$ TEAEs included neutropenia, leukopenia, hypertension and fatigue which had higher incidence in the ramucirumab arm (Table 2). Higher incidence of most common any-grade AESIs (bleeding or hemorrhage [ $42 \%$ vs. $18 \%$ ]; hypertension [ $25 \%$ vs. $6 \%$ ]; proteinuria [17\% vs. $6 \%$ ]; liver injury or failure [17\% vs. $12 \%]$ ]) were observed in the ramucirumab arm versus placebo arm.

A subgroup analysis of East Asian patients in the RAINBOW study indicated that ramucirumab plus paclitaxel significantly improved PFS (5.5 months vs. 2.8 months [HR, $0.628 ; 95 \% \mathrm{CI}, 0.473$ to $0.834 ; \mathrm{p}=0.001])$ and ORR ( $34 \%$ vs. $20 \%, \mathrm{p}=0.0134)$, and had a numerically longer median OS (12.1 months vs. 10.5 months [HR, $0.986 ; 95 \% \mathrm{CI}, 0.727$ to 1.337; $\mathrm{p}=0.929]$ ) compared to placebo [28]. The longer median survival time in East Asian patients compared to non-East Asian patients [28] may be due to the more frequent use of post-study discontinuation treatment by East Asian patients (67\% in both arms combined) compared to non-East Asian patients (37\% in both arms combined). Ramucirumab plus paclitaxel conferred similar improvements in patients $<65$ years and $\geq 65$ years for OS (HRs, 0.753 [95\% CI, 0.604 to 0.939] and 0.861 [ $95 \%$ CI, 0.636 to 1.165], respectively), PFS (HRs, 0.572 [95\% CI, 0.460 to 0.711 ] and 0.673 [95\% CI, 0.506 to 0.894 ], respectively), and ORR (ramucirumab, $28 \%$ and placebo, $14 \%$ vs. ramucirumab, $27 \%$ and placebo, $20 \%$, respectively) [29].

A phase II, multicenter, randomized, double-blind study conducted in the United States investigated ramucirumab plus FOLFOX versus placebo plus FOLFOX in the first-line 


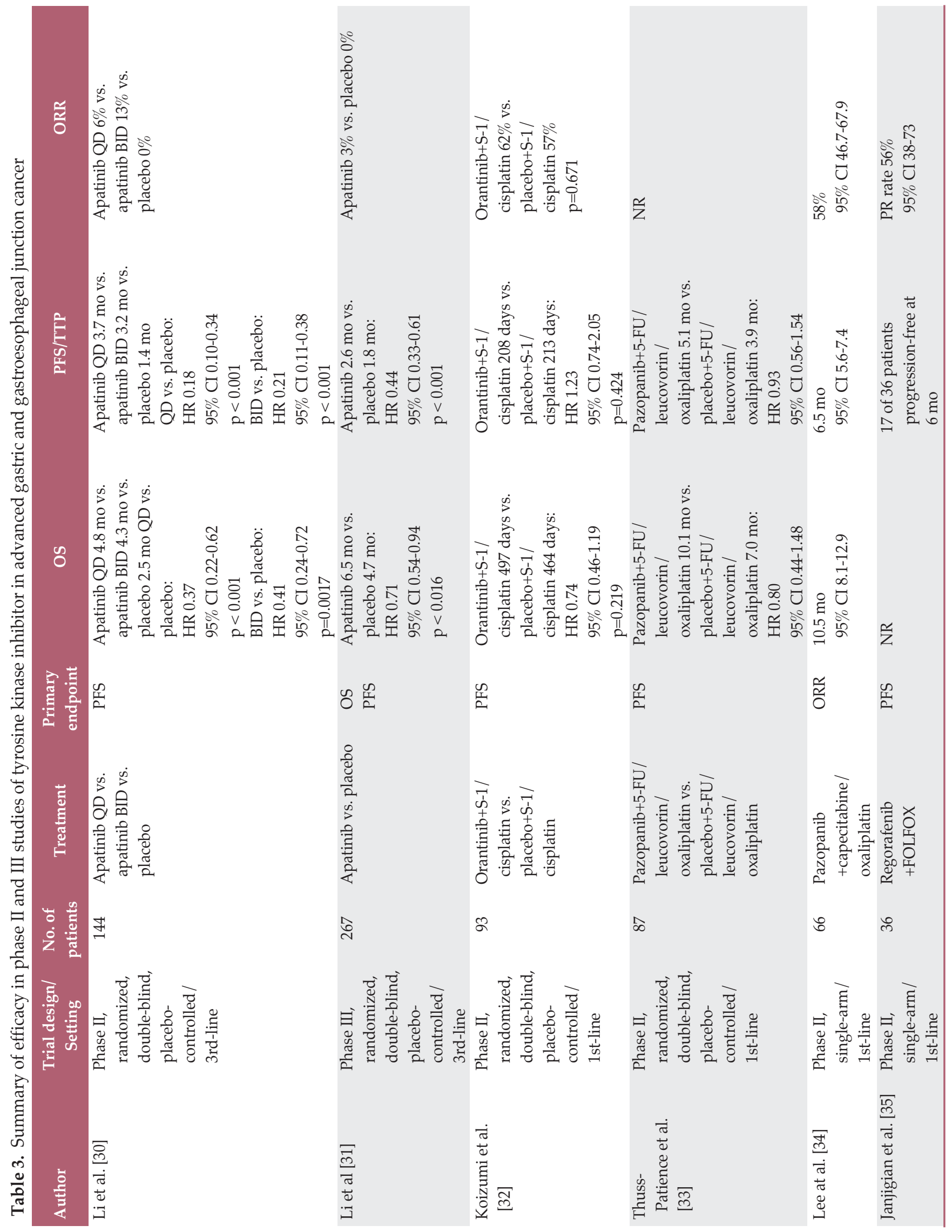



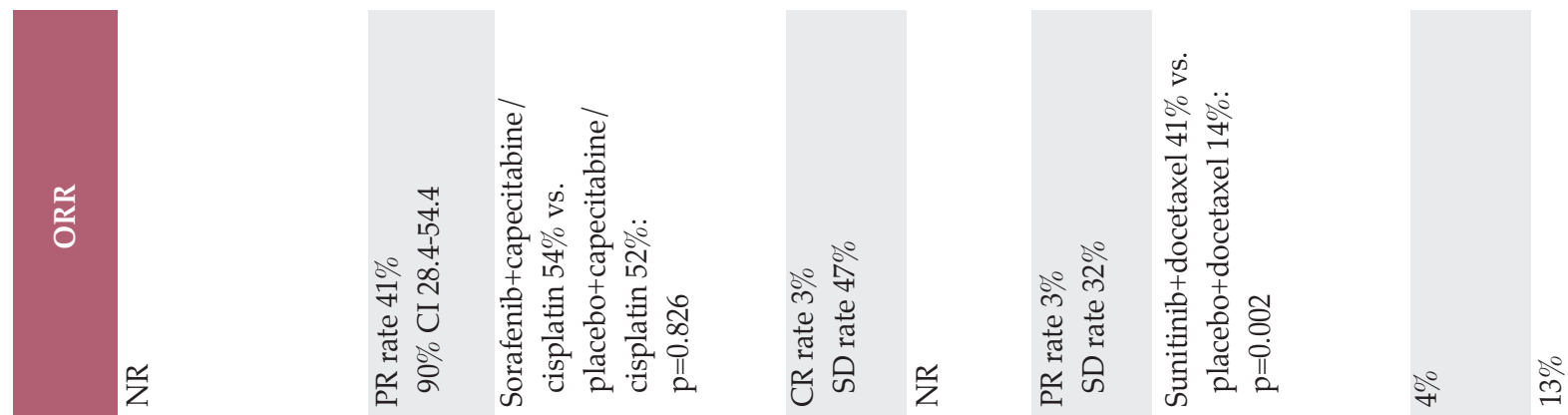

至
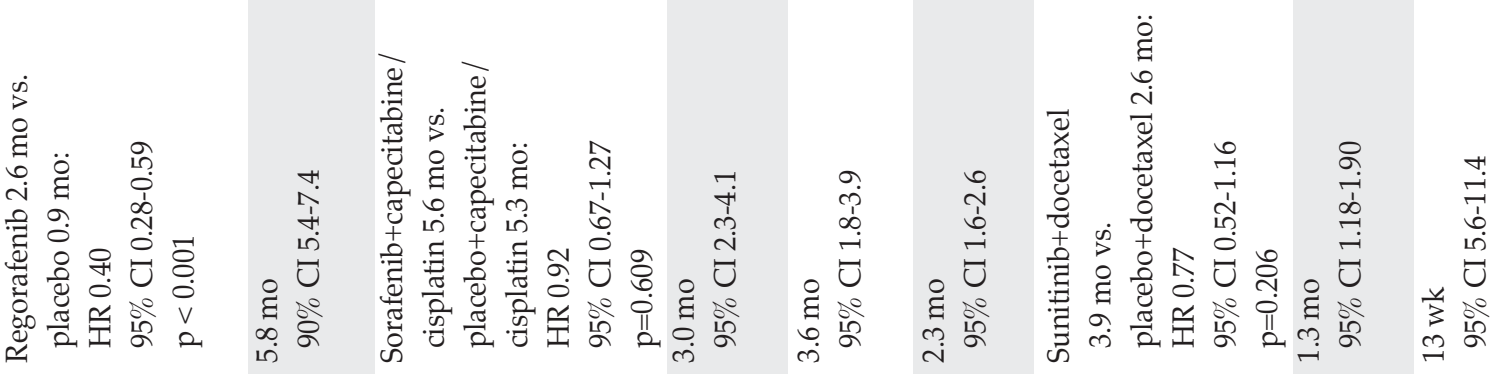

ก)

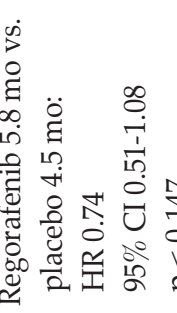

\section{苛

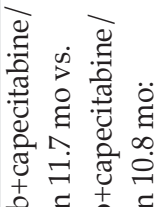

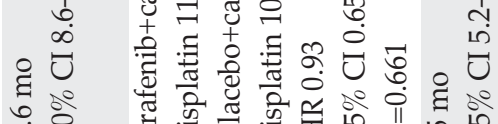

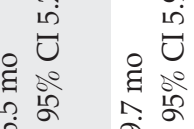

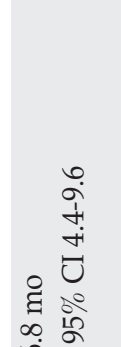

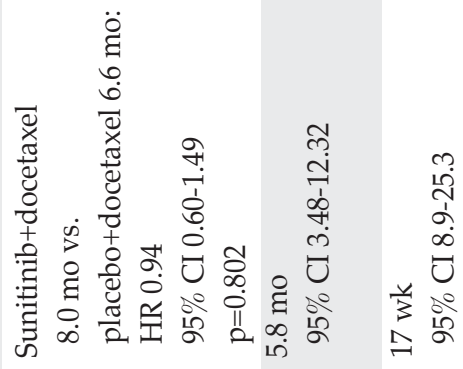

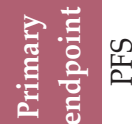

兴 到

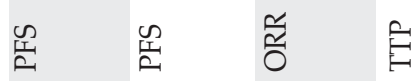

总罂
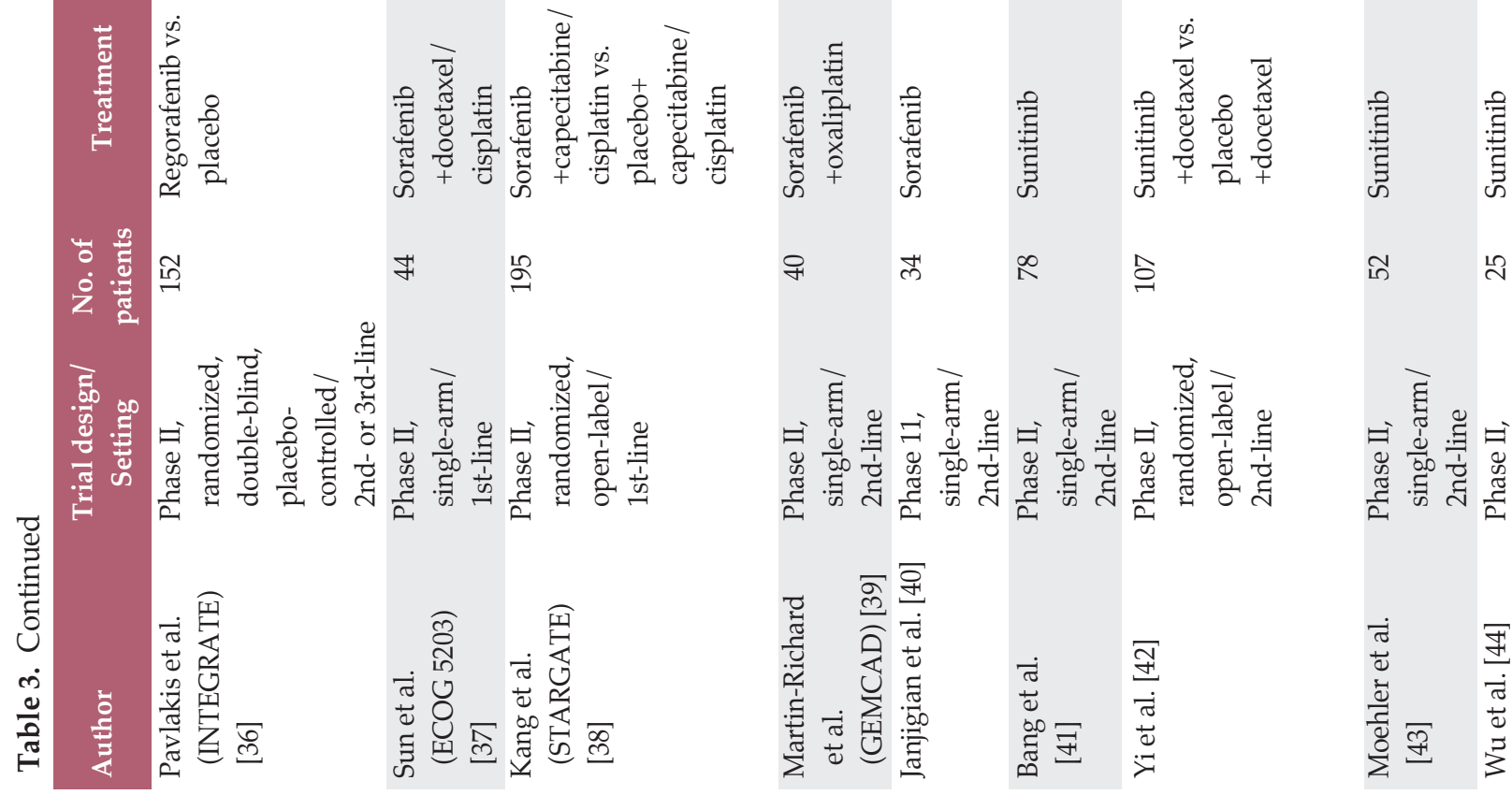

\section{范}

Z $\stackrel{2}{2}$

아 하

$\infty \quad \hat{\sigma}$

กิ นัก

党

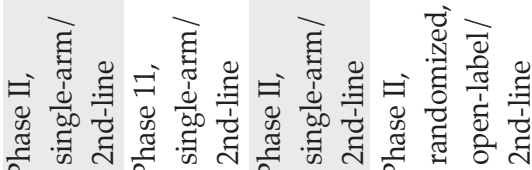

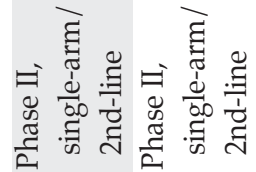

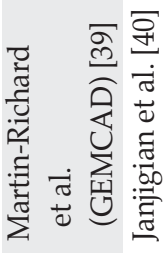

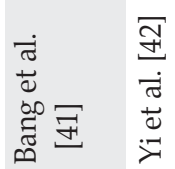

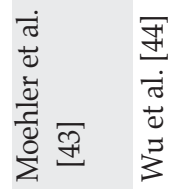



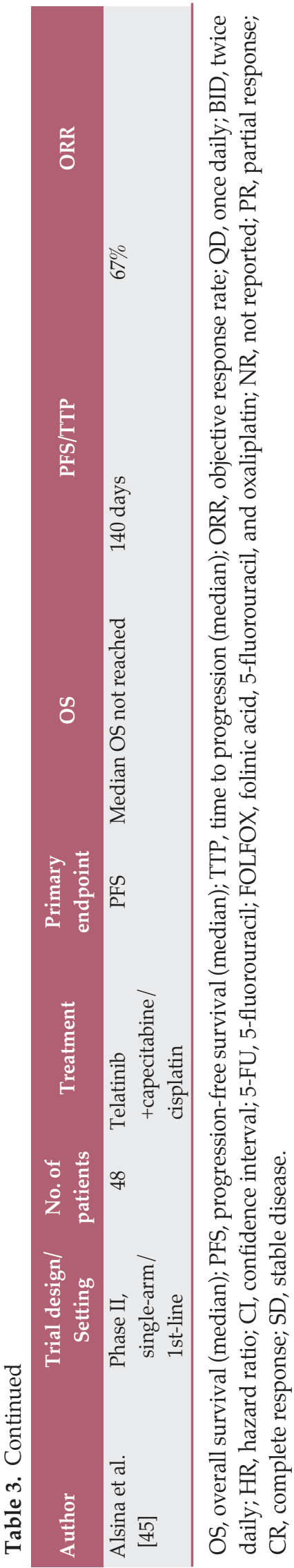

setting in 168 patients with metastatic or locally advanced esophageal, gastroesophageal junction, or gastric cancer [23], and found no significant improvement in median PFS, median OS and ORR (Table 2). However, there was a relatively high proportion of patients discontinued study treatment for reasons other than progressive disease. In exploratory analyses that censored PFS at treatment discontinuation for non-progressive disease the HR for PFS favored ramucirumab plus FOLFOX (HR, 0.76; $\mathrm{p}=0.194)$, mainly in patients with gastric or gastroesophageal junction cancer (median PFS, 9.3 months vs. 7.6 months [HR, $0.53 ; 95 \%$ CI, 0.29 to 0.97 ; $\mathrm{p}=0.036])$. The most common grade $\geq 3$ TEAEs were similar for neutropenia ( $27 \%$ vs. $36 \%)$, fatigue $(18 \%$ vs. $15 \%)$, and neuropathy ( $9 \%$ vs. $11 \%$ ). Grade $\geq 3$ AESIs were uncommon, except hypertension. This study failed to suggest a significant benefit of adding ramucirumab to chemotherapy in the first-line setting. These negative results in the context of the positive results of the REGARD and RAINBOW studies raise questions about the impact of various factors on the efficacy of anti-angiogenic agents that will have to be addressed in future clinical trials, including line of treatment (first- vs. second-line), primary tumor location (esophageal vs. gastric or gastroesophageal junction), and choice of chemotherapy backbone. S5 Table lists the ongoing ramucirumab studies.

Based on the phase III REGARD and RAINBOW studies, current consensus is that ramucirumab plus paclitaxel is a new standard for the second-line treatment of patients with advanced or metastatic gastric or gastroesophageal junction cancer. Ramucirumab as a single agent in the second-line setting is acceptable when paclitaxel or irinotecan cannot be indicated because of severe neutropenia in first-line chemotherapy, neuropathy, or patients' wishes to avoid hair loss.

\section{Efficacy and safety of tyrosine kinase inhibitor therapy}

Tyrosine kinase inhibitors (TKIs) are able to enter cells and interact with the intracellular domain of receptors and intracellular signaling molecules, thereby inhibiting the activation of downstream signaling pathways. Phase II and III studies of apatinib, axitinib, orantinib, pazopanib, regorafenib, sorafenib, sunitinib, telatinib, and vandetanib are summarized in Table 3.

Apatinib is a novel receptor TKI selectively targeting VEGFR-2 with a binding affinity greater than that of other TKIs $[6,46]$. A phase II, randomized, double-blind, placebocontrolled, parallel-arm study investigated placebo versus apatinib $850 \mathrm{mg}$ once daily versus apatinib $425 \mathrm{mg}$ twice daily in the third-line setting in 144 Chinese patients with metastatic or advanced gastric or gastroesophageal junction cancer [30]. To our knowledge, this was the first study to demonstrate improved OS and PFS for a TKI, and the first to show improved median OS median PFS and ORR for both 
doses in the third-line setting (Table 3). Grade 3-4 TEAEs that occurred in greater than $10 \%$ of patients were hand-foot syndrome and hypertension; hematologic toxicities were mostly moderate and grade 3-4 hematologic toxicities were rare.

A phase III, randomized, double-blind, placebo-controlled study investigated apatinib $850 \mathrm{mg}$ once daily versus placebo in the third-line setting, and enrolled 267 patients with advanced gastric cancer [31,47]. Median OS and PFS were significantly improved in the apatinib group compared with the placebo group (Table 3 ). The most common grade 3-4 TEAEs were hand-foot syndrome, proteinuria, and hypertension. Several apatinib studies are currently underway (S5 Table).

Axitinib is a TKI of VEGFR-1, -2, and -3 [48] and has been approved for advanced renal cell carcinoma after failure of one prior systemic therapy. A phase I study investigated axitinib in combination with capecitabine and cisplatin in the first-line setting in 22 patients from Japan and Korea with advanced gastric cancer [49]. Median PFS was 3.8 months, and $36.4 \%$ of patients each had a PR or stable disease; median response duration for patients with PR was 9.1 months. TEAEs included grade 3-4 hypertension (36\% of patients) and decreased appetite and stomatitis (18\% of patients for each); grade 3-4 laboratory abnormalities included neutropenia (36\% of patients) and thrombocytopenia ( $18 \%$ of patients).

Orantinib is a small molecule that competitively inhibits the tyrosine kinase of the receptors for VEGF, basic fibroblast growth factor, and platelet-derived growth factor. A phase II, multicenter, randomized, controlled study investigated orantinib plus S-1 / cisplatin versus S-1/ cisplatin in the firstline setting in 93 chemotherapy-naïve Japanese patients with unresectable or recurrent advanced gastric cancer [32]. Analyses found no significant improvement in median OS, median PFS, and ORR (Table 3). Changes in hemoglobin levels, changes in platelet levels ( $24 \%$ vs. $6 \%$ ), and anorexia ( $18 \%$ vs. $9 \%$ ) were the most common grade $\geq 3$ TEAEs with higher incidence in the oratinib arm, neutropenia had a similar incidence for both treatment groups (S4 Table).

Pazopanib is a TKI that selectively inhibits angiogenesis via VEGFR-1, -2,-3, c-kit, and platelet-derived growth factor receptor (PDGFR) [50] that is approved in advanced renal cell cancer and advanced soft tissue sarcoma in patients who have received prior chemotherapy. Two phase II studies of pazopanib plus fluoropyrimidine and platinum combination in the first-line setting in gastric cancer showed that median OS ranged from 10.1 months to 10.5 months [33,34]. TEAEs included grade $\geq 3$ neutropenia $(15 \%)$, anemia $(11 \%)$, and thrombocytopenia (11\%) [34].

Regorafenib is a VEGFR-2, fibroblast growth factor receptor 2, and PDGFR small-molecule TKI that is approved in metastatic colorectal cancer and in locally advanced, unre- sectable or metastatic gastrointestinal stromal tumor.

A phase II study investigated regorafenib plus FOLFOX in the first-line setting in 36 patients with unresectable or metastatic gastroesophageal junction cancer [35]. A total of 17 of 36 patients were progression-free at 6 months. Another phase II randomized, double-blind, placebo-controlled study (INTEGRATE) investigated regorafenib versus placebo in the second- or third-line setting in 152 patients with refractory or advanced gastroesophageal junction cancer [36,51], and showed significant improvement in median PFS (Table 3). Ongoing studies of regorafenib are listed in S5 Table.

Sorafenib is a TKI with several targets, including Raf- 1 , wild-type and mutant B-Raf, VEGFR-1, -2 , and -3, PDGFR- $\beta$, and Mcl-1 [52]. Sorafenib is approved in hepatocellular carcinoma, renal cell carcinoma, and thyroid carcinoma.

A phase II, single-arm, open-label study (ECOG 5203) investigated sorafenib with docetaxel and cisplatin in 44 chemotherapy-naïve patients with metastatic or advanced gastroesophageal junction cancer and demonstrated a median OS of 13.6 months [37]. A phase I dose-finding study of sorafenib in combination with capecitabine and cisplatin as a first-line treatment in 16 patients with advanced gastric cancer demonstrated a median OS of 14.7 months [53].

A phase II, randomized, open-label study (STARGATE) investigated sorafenib plus capecitabine and cisplatin versus capecitabine and cisplatin in the first-line setting in 195 patients from Korea, China, and Taiwan with metastatic gastric cancer [38]. This study did not demonstrate improvement in median, median, and ORR (Table 3). Of the 51 patients who crossed over to sorafenib alone in the capecitabine and cisplatin arm, there was no objective response and the median PFS was 1.3 months (95\% CI, 1.2 to 1.7). The most common grade 3-4 TEAEs included neutropenia, febrile neutropenia, and palmar-plantar erythrodysesthesia syndrome (S4 Table).

A phase II, multicenter, single-arm study in the secondline setting investigated sorafenib plus oxaliplatin in 40 patients with progressive gastric adenocarcinoma after fluoropyrimidine and cisplatin treatment [39]. Median OS and PFS were 6.5 months and 3.0 months, respectively; the CR rate was $2.5 \%$, and $47.2 \%$ of patients achieved stable disease. TEAEs included grade $3-4$ asthenia (18\%), neutropenia (10\%), and thrombocytopenia (8\%). A phase II, single arm study investigated sorafenib in 34 patients with advanced esophageal and gastroesophageal junction cancer in the second line setting [40]. Median OS and PFS were 9.7 months and 3.6 months, respectively. The most common grade 3 TEAEs included hand-foot reaction (12\%) and fatigue $(6 \%)$.

Sunitinib is a multi-targeted TKI of VEGFR-1, -2 , and -3 , PDGFR- $\alpha$ and $-\beta$, and several other receptor tyrosine kinases [54]. Sunitinib is approved for advanced renal cell carcinoma, gastrointestinal stromal tumor, and pancreatic neuroen- 
docrine tumors. Multiple phase II studies of sunitinib in gastric cancer in the second-line setting showed that median OS ranged from 5.8 months to 8.0 months, and median PFS/TTP ranged from 1.3 months to 3.9 months [41-43]. TEAEs included grade $\geq 3$ thrombocytopenia (35\% of patients), neutropenia ( $29 \%$ of patients), and anemia (17\% of patients) [41]. Wu et al. [44] reported a median PFS of 7 weeks and median OS of 17 weeks in a single arm phase II studies conducted in patients with relapsed/refractory oesophageal or gastrooesophageal cancer with anemia, thrombocytopenia, and leukopenia reported as the most common TEAEs.

Telatinib is a TKI of the VEGFR family, PDGFR, and KIT receptor tyrosine kinases. A phase II multicenter study investigated telatinib in combination with capecitabine and cisplatin in the first-line setting in 48 patients with advanced gastric or gastroesophageal junction cancer [45]. Median OS was not reached, and the most common toxicity was grade 1-2 fatigue.

Vandetanib is a TKI of VEGFR-2, VEGFR-3, EGFR, and RET kinase [55] that is approved for thyroid cancer. A phase I study investigated vandetanib plus oxaliplatin and docetaxel in the first-line setting in nine patients with advanced gastric or gastroesophageal junction cancer and demonstrated a median OS of 5.5 months [56].

\section{Quality of life}

Patient-reported QoL for phase II and III studies of antiangiogenic agents are summarized in S6 Table. In the AVAGAST study, mean European Organisation for Research and Treatment of Cancer Quality of Life Questionnaire C30 (EORTC QLQ-C30) global health status scores were similar over time in both treatment groups [18]. In the REGARD study, more patients in the ramucirumab group than in the placebo group reported stable or improved EORTC QLQC30 global health status scores, although this difference was not significant $(\mathrm{p}=0.23)$; however, median time to performance status deterioration was 5.1 months for ramucirumab and 2.4 months for placebo (HR, 0.59 ; $95 \%$ CI, 0.41 to 0.83 ; $\mathrm{p}=0.002$ ) [21]. In the RAINBOW study, for all EORTC QLQC30 and EuroQoL five-dimension, three-level health status questionnaire (EQ-5D-3L) parameters, and at all on-therapy assessment times, the proportion of patients reporting improved or stable scores from baseline to discontinuation was numerically greater for the ramucirumab group [57]. In the phase II, randomized, placebo-controlled, parallel-arm study of apatinib, which investigated placebo versus apatinib $850 \mathrm{mg}$ once daily versus apatinib $425 \mathrm{mg}$ twice daily, there were no significant differences in EORTC QLQ-C30 different parameters between the three treatment groups over the course of the treatment [30]. In the phase II open-label study of sunitinib, EORTC QLQ-C30 global health status scores were mostly maintained by sunitinib treatment during the first three cycles of the study, although the domains of diarrhea and reflux symptoms were noticeably worse compared to baseline [41]. At patients' last evaluation (end of treatment or withdrawal from the study), noticeable deterioration was observed in most scales and measures of the EORTC QLQC30 and the stomach cancer specific questionnaire (QLQSTO22) compared to the baseline, whereas the domains for perceived financial difficulties, body image, and hair loss did not change noticeably [41].

\section{Biomarkers}

Identification of tumors most sensitive to therapy could improve therapeutic approaches and provide insight into mechanisms of resistance and further refine anti-angiogenic therapy [58-60]. In two phase II studies of bevacizumab, neuropilin-2 mRNA significantly inversely correlated with PFS [17], while high VEGF-A correlated with longer OS and high VEGFR-2 correlated with shorter PFS [61]. In the phase III AVAGAST study, high VEGF-A (HR, $0.72 ; 95 \%$ CI, 0.57 to 0.93 ) and low neuropilin- $1 \mathrm{HR}, 0.75 ; 95 \% \mathrm{CI}, 0.59$ to 0.97$)$ showed a trend for improved OS; however, these findings were only found to be statistically significant in patients from non-Asian regions [61]. A reassessment of VEGF-A as a candidate predictive biomarker for bevacizumab in 13 trials and seven indications showed that VEGF-A level was not a robust predictive biomarker for bevacizumab activity, and that stratification of patients based on a single baseline measurement of VEGF-A is unlikely to be implemented in clinical practice [62]. An assessment on the role of angiopoietin-2 as a prognostic biomarker based on the AVAGAST study data demonstrated that baseline angiopoietin-2 levels correlate with liver metastasis across ethnic groups and that angiopoietin-2 may be a predictive biomarker for gastric cancer [63]. In the phase III REGARD study, analysis of biomarkers showed that HER2; VEGFR-1, -2, -3; and VEGF-C, -D were not markers for ramucirumab efficacy [64]. In multiple phase II studies of sunitinib, tumor VEGF-C expression was associated with significantly shorter median PFS (1.23 months vs. 2.86 months, log-rank $\mathrm{p}=0.0119)$ [43], and high sKIT $(\mathrm{p}=0.0081)$ and low VEGF-C $(\mathrm{p}=0.0326)$ were significantly associated with clinical benefit [41]. Despite these initial observations, there have been no confirmatory studies to date. 


\section{Discussion}

Angiogenesis plays a key role in the development and progression of gastric and gastroesophageal junction cancer and tumor vasculature enables malignant cells to escape from the primary site and establish distant metastasis elsewhere. Hence, agents that specifically target angiogenesis in gastric cancer are desirable.

Preclinical studies indicate that inhibition of angiogenesis signaling pathways may be an important therapeutic approach. Few clinical trials using anti-angiogenic agents have so far shown a significant survival benefit, possibly due to disease heterogeneity, tumor microenvironment and the complex relationship between tumors and their surrounding vasculature and stroma, a lack of driver genes, and difficulties in identifying a reliable predictive biomarker.

Due to its increased expression in most human cancers and association with increased tumor microvessel density and poor prognosis, the VEGF pathway has been the leading target for antiangiogenic therapy. Several tumors are sensitive to VEGF inhibitors; however, the degree of noted benefits in clinical trials has been variable across tumor sites and the different VEGF inhibitors. The underlying molecular biology in clear cell renal carcinoma, characterized by inactivation of the von-Hippel Lindau gene which results in hypoxiainducible factor upregulation and VEGF induction, may account for the high sensitivity of this tumor to anti-VEGF therapy. Generally, however, the reasons of the differences in sensitivity among tumor types and individual anti-VEGF agents are difficult to explain [65]. Based on results of randomized studies, gastro-oesophageal cancer has been considered, together with colorectal cancer, glioma, breast cancer and lung cancer, to be an antiangiogenic drug-sensitive cancer [65].

This systematic review of published literature found that ramucirumab and apatinib are the only anti-angiogenic agents that significantly improve survival of patients with advanced gastric cancer. The published literature also indicates that anti-angiogenic agents are generally well tolerated, and do not appear to impair QoL. No biomarkers of response to anti-angiogenic agents were identified in the clinical setting, and no anti-angiogenic agent was found to have a survival benefit in the first-line setting, possibly due to differences in tumor biology and subsequent treatment between the first- and second-line settings, or limitations of study designs and patient selection.

While only a few individual clinical trials have shown a significant survival benefit, a systematic review and metaanalysis of 22 randomized clinical trials with a total of 7,022 advanced gastric cancer patients demonstrated OS benefit for anti-angiogenic agents (HR, 0.759; 95\% CI, 0.655 to 0.880 ; $\mathrm{p}<0.001$ ) [66]. Another systematic review and meta-analysis found that addition of anti-angiogenic agents improved OS in metastatic gastroesophageal junction cancer (HR, 0.80; 95\% CI, 0.74 to $0.87 ; \mathrm{p}<0.00001$ ) [67]. OS was not improved in the first-line setting (HR, $0.91 ; 95 \% \mathrm{CI}, 0.80$ to 1.05$)$ but was improved in second- and third-line settings (HR, 0.74; 95\% CI, 0.66 to 0.82 ) [67]. These findings differ from the outcomes of individual studies, possibly due to poor study design or patient selection.

Geographic differences in gastric cancer are evident between patients from Asian regions and patients from Western regions with respect to prevalence, clinicopathologic features, and preference in treatment patterns [1,68-72]. Subgroup analyses of individual studies have provided mixed results on the benefit of anti-angiogenic agents in patients from East Asia, which has approximately half of the total world gastric cancer, and the highest estimated mortality rates for gastric cancer [1]. The RAINBOW study showed that the improvements in PFS and ORR in East Asian patients were consistent with the improvements in non-East Asian patients $[22,28]$. In support of this finding, a systematic review and meta-analysis by Chan et al. [67] demonstrated OS benefit for anti-angiogenic agents in the second-line setting for gastric cancer in all regions (Europe, Asia, and PanAmerica). In the AVAGAST study, Pan-American patients showed a survival benefit whereas Asian patients had no benefit, and European patients had intermediate results [18]. A higher proportion of patients in East Asia receive secondline or further-line therapy compared to the United States and Europe, which may have contributed to the longer median survival observed in phase III studies conducted in East Asia [73-75]. In addition, a trend for regional differences in the AVAGAST study were noted in PFS (Asia HR, 0.92; Europe HR, 0.71; Pan-America HR, 0.65) [18], suggesting potential differences in the benefits of bevacizumab for Asian patients compared to European or Pan-American patients.

With the emergence of effective second or later lines of therapy, there is increasing debate on the use of PFS as a surrogate endpoint for frontline systemic therapy in gastric cancer. For instance, AVAGAST would have been a positive trial if PFS had been used as the endpoint rather than OS [18]. In an analysis of 36 randomized studies in advanced gastric cancer, improvements in PFS / TTP strongly correlated with improvements in OS [76]. A meta-analysis of 4,069 patients in 20 randomized studies of gastric cancer showed the rank correlation coefficient between PFS and OS was 0.853 (95\% CI, 0.852 to 0.854 ). Although the treatment effects on PFS and OS were only moderately correlated, the validity of PFS as a surrogate endpoint for OS in advanced/recurrent gastric cancer could not be confirmed [77]. Therefore, the ongoing phase III RAINFALL study of ramucirumab in combination with capecitabine and cisplatin as first-line therapy in 
patients with gastric cancer selected PFS as the primary endpoint.

In addition to survival, QoL and quality-adjusted survival should be fundamental therapeutic objectives for gastric cancer. The impact of treatment-related toxicity on QoL has been scantly reported for patients with gastric cancer. A number of barriers may hinder assessment of QoL; the evaluation of QoL may not be accurately reflected by self-reported questionnaires, and disease deterioration may also lead to a low compliance rate. Nonetheless, there is emerging evidence that chemotherapy provides substantial improvement in QoL for patients with gastric and gastroesophageal junction cancer [78] as evident for ramucirumab [21,22,79].

Some preclinical evidence indicates that certain chemotherapy regimens, most notably taxanes, rapidly induce the release of proangiogenic circulating endothelial progenitor cells which can be abrogated by treatment with antiangiogenic agents [80]. As presented in this systematic review, a number of phase II and III clinical trials have assessed antiangiogenic agents in combination with conventional chemotherapy in treating patients with advanced gastric cancer and gastroesophageal junction adenocarcinoma. These trials included the use of a fluoropyrimidine-platinum doublet with or without a third chemotherapeutic drug in the firstline setting, and the use of taxanes or irinotecan as a chemotherapy backbone in the second-line setting. Given the heterogeneity in patient profiles, study size, the various combinations and pharmacodynamics / pharmacokinetic properties of the chemotherapeutic agents, and the nature of anti-angiogenic agents, we could not make any firm conclusions. Among trials that examined the use of taxanes (paclitaxel or docetaxel) as a chemotherapy partner with an antiangiogenic agent in advanced gastric cancer, RAINBOW (paclitaxel plus ramucirumab as second-line therapy for advanced gastric cancer) was the first and only study to demonstrate survival benefits. Therefore, a chemotherapy backbone does not appear to be the sole factor influencing the efficacy of anti-angiogenic agents.

The awareness that crosstalk exists between angiogenic and oncogenic signaling pathways has prompted the investigation of combinations of antiangiogenics with targeted agents, in an attempt to improve clinical outcomes. The addition of the anti-VEGF antibody bevacizumab or ramucirumab to the anti-HER2 antibody trastuzumab on top of standard chemotherapy is being investigated in HER2-positive metastatic cancer patients (S5 Table). Also, the combination of antiangiogenic agents with immunotherapy is a relatively recent area of great interest. A synergistic antitumor effect was demonstrated when the VEGFR-2 and PD-1 were simultaneously blocked in a preclinical murine colon cancer model [81]. Preclinical data also demonstrated that an antiVEGFR-2 antibody can reprogram the tumor microenviron- ment away from immunosuppression [82]. Following these lines of investigations, two ongoing phase I studies explore the combination of ramucirumab with the anti-PD-1 antibody pembrolizumab and with the anti-PD-L1 antibody durvalumab (S5 Table) in cohorts of patients with different tumor types including gastric/gastroesophageal junction adenocarcinoma.

Predictive biomarkers for the clinical outcome of antiangiogenic agents have not been identified in the treatment of gastric and gastroesophageal junction cancer. Nonetheless, a retrospective analysis of biomarkers in AVAGAST showed that plasma VEGF-A and tumor neuropilin-1 were promising biomarker candidates for predicting clinical outcome in patients with advanced gastric cancer treated with bevacizumab [61]. Due to the lack of predictive biomarkers, the current recommended study design for investigating antiangiogenic agents would be a conventional approach, that is enrolling patients based on clinico-pathologic characteristics coupled with prespecified analysis of biomarker defined subgroups, where the biomarker is chosen based on the biological rationale of the mechanism of action of the molecule. Such a design would incorporate both all-comers and targetenriched designs. This would ensure an incorrect conclusion that the drug is not effective does not occur. In addition, by identifying molecular traits relevant to the action of the therapeutic agents, specific subgroups of patients can be identified and studied further.

A recent study conducted by the Asian Cancer Research Group used gene expression data to describe four molecular subtypes of gastric cancer linked to distinct patterns of molecular alterations, disease progression, and prognosis [83]. The subtypes were validated in independent cohorts and are expected to guide future molecular screening and therapeutic development in gastric cancer [83]. A number of new innovative study designs, such as basket study or adaptive trial, are being developed using information gained from high-throughput molecular profiling and may bring a new revolution for drug development.

In studies of single agents, there is evidence to suggest that the safety profiles of anti-angiogenic antibodies that target the VEGF/VEGFR-2 differ from those that target multiple receptor tyrosine kinases. The most frequent grade $\geq 3$ TEAEs are summarized in S4 Table. Compared with antiangiogenic antibodies that target the VEGF/VEGFR-2 [18,19, 21,22], multi-target TKIs are generally associated with a higher incidence of hematologic TEAEs, especially neutropenia, leukopenia, thrombocytopenia, elevations in liver enzymes and hand-foot syndrome [30,37,39,41-43]. On the other hand, the incidence of fatigue was relatively high with ramucirumab and several of the multi-target TKIs [21,22,37, $41,43]$, with the exception of bevacizumab $[18,19]$. While the toxicity profiles of ramucirumab and apatinib differ, neither 
appear to be better than the other in terms of toxicity $[21,47]$. One advantage of oral TKIs is that they have no risk of infusion-related reaction, although infusion-related reactions appeared to be rare with ramucirumab [21,22]. Overall, these data suggest that anti-angiogenic agents that specifically target the VEGF / VEGFR-2 will have a better safety profile than the multi-target TKIs.

There are inherent limitations to this systematic review that should be considered. During the study selection process, three articles were identified outside of the original search strategy for full manuscript review, which were included in the final set of 189 articles; the identification of articles from outside the original literature search suggests some limitations in the search strategy. In addition, the current review was primarily descriptive, providing an overview of efficacy, safety, QoL, and biomarkers in the relevant studies. Furthermore, by excluding articles written in languages other than English, it is possible our systematic review missed some potential additional insights.

In conclusion, ramucirumab, either as monotherapy or in combination with paclitaxel, and apatinib are the only antiangiogenic agents to significantly improve survival of patients with previously treated advanced gastric cancer.
There are no predictive biomarkers for response of gastric cancer to anti-angiogenic agents available for use in the clinical setting.

\section{Electronic Supplementary Material}

Supplementary materials are available at Cancer Research and Treatment website (http:// www.e-crt.org).

\section{Conflicts of Interest}

Roberto Carlesi, Rebecca Cheng, Jongseok Kim, and Mauro Orlando are employees of Eli Lilly and Company. Winnie Yeo has received honorarium from Eli Lilly and Company. All remaining authors have declared no conflicts of interest.

\section{Acknowledgments}

This review was sponsored by Eli Lilly and Company. Medical writing support was provided by Andrew Sakko, and editorial support was provided by Noelle Gasco, of inVentiv Health Clinical and funded by Eli Lilly and Company.

\section{References}

1. Ferlay J, Soerjomataram I, Ervik M, Dikshit R, Eser S, Mathers C, et al. GLOBOCAN 2012 v1.0, Cancer incidence and mortality worldwide: IARC CancerBase No. 11 [Internet]. Lyon: International Agency for Research on Cancer; 2013 [cited 2015 Nov 25]. Available from: http://globocan.iarc.fr.

2. van Hagen $P$, Hulshof MC, van Lanschot JJ, Steyerberg EW, van Berge Henegouwen MI, Wijnhoven BP, et al. Preoperative chemoradiotherapy for esophageal or junctional cancer. $\mathrm{N}$ Engl J Med. 2012;366:2074-84.

3. Wagner AD, Grothe W, Haerting J, Kleber G, Grothey A, Fleig WE. Chemotherapy in advanced gastric cancer: a systematic review and meta-analysis based on aggregate data. J Clin Oncol. 2006;24:2903-9.

4. Kang JH, Lee SI, Lim DH, Park KW, Oh SY, Kwon HC, et al. Salvage chemotherapy for pretreated gastric cancer: a randomized phase III trial comparing chemotherapy plus best supportive care with best supportive care alone. J Clin Oncol. 2012;30:1513-8.

5. Kim SE, Shim KN, Jung SA, Yoo K, Lee JH. The clinicopathological significance of tissue levels of hypoxia-inducible factor-1alpha and vascular endothelial growth factor in gastric cancer. Gut Liver. 2009;3:88-94.

6. Tian S, Quan H, Xie C, Guo H, Lu F, Xu Y, et al. YN968D1 is a novel and selective inhibitor of vascular endothelial growth factor receptor- 2 tyrosine kinase with potent activity in vitro and in vivo. Cancer Sci. 2011;102:1374-80.

7. Hicklin DJ, Ellis LM. Role of the vascular endothelial growth factor pathway in tumor growth and angiogenesis. J Clin Oncol. 2005;23:1011-27.

8. Faivre S, Demetri G, Sargent W, Raymond E. Molecular basis for sunitinib efficacy and future clinical development. Nat Rev Drug Discov. 2007;6:734-45.

9. Suri C, Jones PF, Patan S, Bartunkova S, Maisonpierre PC, Davis $S$, et al. Requisite role of angiopoietin-1, a ligand for the TIE2 receptor, during embryonic angiogenesis. Cell. 1996;87: 1171-80.

10. Quatrale AE, Porcelli L, Gnoni A, Numico G, Paradiso A, Azzariti A. New vascular disrupting agents in upper gastrointestinal malignancies. Curr Med Chem. 2014;21:1039-49.

11. Zimna A, Kurpisz M. Hypoxia-inducible factor-1 in physiological and pathophysiological angiogenesis: applications and therapies. Biomed Res Int. 2015;2015:549412.

12. Moher D, Liberati A, Tetzlaff J, Altman DG; PRISMA Group. Preferred reporting items for systematic reviews and metaanalyses: the PRISMA statement. BMJ. 2009;339:b2535.

13. Shah MA, Jhawer M, Ilson DH, Lefkowitz RA, Robinson E, Capanu M, et al. Phase II study of modified docetaxel, cisplatin, and fluorouracil with bevacizumab in patients with metastatic gastroesophageal adenocarcinoma. J Clin Oncol. 2011;29:868-74. 
14. Shah MA, Ramanathan RK, Ilson DH, Levnor A, D'Adamo D, O'Reilly E, et al. Multicenter phase II study of irinotecan, cisplatin, and bevacizumab in patients with metastatic gastric or gastroesophageal junction adenocarcinoma. J Clin Oncol. 2006; 24:5201-6.

15. Enzinger PC, Abrams TA, Chan JA, McCleary NJ, Zheng H, Kwak EL, et al. Multicenter phase 2: capecitabine (CAP) + oxaliplatin $(\mathrm{OX})+$ bevacizumab (BEV) + trastuzumab (TRAS) for patients (pts) with metastatic esophagogastric cancer (MEGCA). J Clin Oncol. 2015;33 Suppl:Abstr 4038.

16. El-Rayes BF, Zalupski M, Bekai-Saab T, Heilbrun LK, Hammad N, Patel B, et al. A phase II study of bevacizumab, oxaliplatin, and docetaxel in locally advanced and metastatic gastric and gastroesophageal junction cancers. Ann Oncol. 2010;21:1999-2004.

17. Uronis HE, Bendell JC, Altomare I, Blobe GC, Hsu SD, Morse MA, et al. A phase II study of capecitabine, oxaliplatin, and bevacizumab in the treatment of metastatic esophagogastric adenocarcinomas. Oncologist. 2013;18:271-2.

18. Ohtsu A, Shah MA, Van Cutsem E, Rha SY, Sawaki A, Park $\mathrm{SR}$, et al. Bevacizumab in combination with chemotherapy as first-line therapy in advanced gastric cancer: a randomized, double-blind, placebo-controlled phase III study. J Clin Oncol. 2011;29:3968-76.

19. Shen L, Li J, Xu J, Pan H, Dai G, Qin S, et al. Bevacizumab plus capecitabine and cisplatin in Chinese patients with inoperable locally advanced or metastatic gastric or gastroesophageal junction cancer: randomized, double-blind, phase III study (AVATAR study). Gastric Cancer. 2015;18:168-76.

20. Cunningham D, Smyth E, Stenning S, Stevenson L, Robb C, Allum $W$, et al. Peri-operative chemotherapy \pm bevacizumab for resectable gastro-oesophageal adenocarcinoma: results from the UK Medical Research Council randomised ST03 trial (ISRCTN 46020948). Eur J Cancer. 2015;51 Suppl 3:S400.

21. Fuchs CS, Tomasek J, Yong CJ, Dumitru F, Passalacqua R, Goswami C, et al. Ramucirumab monotherapy for previously treated advanced gastric or gastro-oesophageal junction adenocarcinoma (REGARD): an international, randomised, multicentre, placebo-controlled, phase 3 trial. Lancet. 2014;383: 31-9.

22. Wilke H, Muro K, Van Cutsem E, Oh SC, Bodoky G, Shimada $\mathrm{Y}$, et al. Ramucirumab plus paclitaxel versus placebo plus paclitaxel in patients with previously treated advanced gastric or gastro-oesophageal junction adenocarcinoma (RAINBOW): a double-blind, randomised phase 3 trial. Lancet Oncol. 2014;15:1224-35.

23. Yoon HH, Bendell JC, Braiteh FS, Firdaus I, Philip PA, Cohn $\mathrm{AL}$, et al. Ramucirumab (RAM) plus FOLFOX as front-line therapy $(\mathrm{Rx})$ for advanced gastric or esophageal adenocarcinoma (GE-AC): randomized, double-blind, multicenter phase 2 trial. J Clin Oncol. 2014;32 Suppl:Abstr 4004.

24. Okines AF, Langley RE, Thompson LC, Stenning SP, Stevenson L, Falk S, et al. Bevacizumab with peri-operative epirubicin, cisplatin and capecitabine (ECX) in localised gastrooesophageal adenocarcinoma: a safety report. Ann Oncol. 2013;24:702-9.
25. Enzinger PC, McCleary NJ, Zheng H, Abrams TA, Yurgelun MB, Azzoli CG, et al. Multicenter double-blind randomized phase II: FOLFOX + ziv-aflibercept/ placebo for patients (pts) with chemo-naive metastatic esophagogastric adenocarcinoma (MEGA). J Clin Oncol. 2016;34 Suppl 4S:Abstr 4.

26. Spratlin JL, Cohen RB, Eadens M, Gore L, Camidge DR, Diab $\mathrm{S}$, et al. Phase I pharmacologic and biologic study of ramucirumab (IMC-1121B), a fully human immunoglobulin G1 monoclonal antibody targeting the vascular endothelial growth factor receptor-2. J Clin Oncol. 2010;28:780-7.

27. Fuchs CS, Tomasek J, Cho JY, Tomasello G, Goswami C, Vieira dos Santos L, et al. REGARD: a phase 3, randomized, doubleblind trial of ramucirumab (RAM) and best supportive care (BSC) versus placebo (PL) and BSC in the treatment of metastatic gastric or gastroesophageal junction (GEJ) adenocarcinoma following disease progression (PD) on first-line platinum- and/or fluoropyrimidine-containing combination therapy: age subgroup analysis. J Clin Oncol. 2014;32 Suppl: Abstr 4057.

28. Muro K, Oh SC, Shimada Y, Lee KW, Yen CJ, Chao Y, et al. Subgroup analysis of East Asians in RAINBOW: A phase 3 trial of ramucirumab plus paclitaxel for advanced gastric cancer. J Gastroenterol Hepatol. 2016;31:581-9.

29. Muro K, Bodoky G, Cesas A, Chao Y, Clingan P, Hironaka S, et al. RAINBOW: a global, phase 3, double-blind study of ramucirumab (RAM) plus paclitaxel (PTX) versus placebo (PL) plus PTX in the treatment of advanced gastric and gastroesophageal junction (GEJ) adenocarcinoma following disease progression on first-line platinum- and fluoropyrimidine-containing combination therapy: an age-group analysis. J Clin Oncol. 2015;33 Suppl 3:Abstr 11.

30. Li J, Qin S, Xu J, Guo W, Xiong J, Bai Y, et al. Apatinib for chemotherapy-refractory advanced metastatic gastric cancer: results from a randomized, placebo-controlled, parallel-arm, phase II trial. J Clin Oncol. 2013;31:3219-25.

31. Li J, Qin S, Xu J, Xiong J, Wu C, Bai Y, et al. Randomized, double-blind, placebo-controlled phase III trial of apatinib in patients with chemotherapy-refractory advanced or metastatic adenocarcinoma of the stomach or gastroesophageal junction. J Clin Oncol. 2016;34:1448-54.

32. Koizumi W, Yamaguchi K, Hosaka H, Takinishi Y, Nakayama N, Hara T, et al. Randomised phase II study of S-1/ cisplatin plus TSU-68 vs S-1/ cisplatin in patients with advanced gastric cancer. Br J Cancer. 2013;109:2079-86.

33. Thuss-Patience PC, Al-Batran SE, Siveke JT, Homann N, Malfertheiner P, Glaeser D, et al. Pazopanib and 5-FU/oxaliplatin as first-line treatment in advanced gastric cancer: PaFLO, a randomized phase II study from the AIO (Arbeitsgemeinschaft Internistische Onkologie). J Clin Oncol. 2015;33 Suppl:Abstr 4033.

34. Lee MY, Kim ST, Lee J, Park SH, Park YS, Lim HY, et al. Prospective phase II trial of pazopanib plus CapeOX (capecitabine and oxaliplatin) in previously untreated patients with advanced gastric cancer. J Clin Oncol. 2015;33 Suppl: Abstr 4049.

35. Janjigian YY, Ku GY, Chou JF, Capanu M, Siebel M, Chalasani 
SB, et al. Phase II study of FOLFOX plus regorafenib (REGO) in patients with unresectable or metastatic esophagogastric (EG) cancer. J Clin Oncol. 2015;33 Suppl:Abstr 4053.

36. Pavlakis N, Sjoquist KM, Martin AJ, Tsobanis E, Yip S, Kang YK, et al. Regorafenib for the Treatment of Advanced Gastric Cancer (INTEGRATE): a multinational placebo-controlled phase II trial. J Clin Oncol. 2016;34:2728-35.

37. Sun W, Powell M, O'Dwyer PJ, Catalano P, Ansari RH, Benson AB 3rd. Phase II study of sorafenib in combination with docetaxel and cisplatin in the treatment of metastatic or advanced gastric and gastroesophageal junction adenocarcinoma: ECOG 5203. J Clin Oncol. 2010;28:2947-51.

38. Kang Y, Lee KH, Shen L, Yeh K, Hong YS, Park YI, et al. Randomized phase II study of capecitabine and cisplatin with or without sorafenib in patients with metastatic gastric cancer: STARGATE study. Ann Oncol. 2014;25(Suppl 4):iv210.

39. Martin-Richard M, Gallego R, Pericay C, Garcia Foncillas J, Queralt B, Casado E, et al. Multicenter phase II study of oxaliplatin and sorafenib in advanced gastric adenocarcinoma after failure of cisplatin and fluoropyrimidine treatment. A GEMCAD study. Invest New Drugs. 2013;31:1573-9.

40. Janjigian YY, Vakiani E, Ku GY, Herrera JM, Tang LH, Bouvier $\mathrm{N}$, et al. Phase II trial of sorafenib in patients with chemotherapy refractory metastatic esophageal and gastroesophageal (GE) junction cancer. PLoS One. 2015;10:e0134731.

41. Bang YJ, Kang YK, Kang WK, Boku N, Chung HC, Chen JS, et al. Phase II study of sunitinib as second-line treatment for advanced gastric cancer. Invest New Drugs. 2011;29:1449-58.

42. Yi JH, Lee J, Park SH, Park JO, Yim DS, Park YS, et al. Randomised phase II trial of docetaxel and sunitinib in patients with metastatic gastric cancer who were previously treated with fluoropyrimidine and platinum. Br J Cancer. 2012;106: 1469-74.

43. Moehler M, Mueller A, Hartmann JT, Ebert MP, Al-Batran SE, Reimer $\mathrm{P}$, et al. An open-label, multicentre biomarker-oriented AIO phase II trial of sunitinib for patients with chemo-refractory advanced gastric cancer. Eur J Cancer. 2011;47:1511-20.

44. Wu C, Mikhail S, Wei L, Timmers C, Tahiri S, Neal A, et al. A phase II and pharmacodynamic study of sunitinib in relapsed/refractory oesophageal and gastro-oesophageal cancers. Br J Cancer. 2015;113:220-5.

45. Alsina M, Ko AH, Garcia De Paredes M, Rivera F, Schwartzberg LS, Fattaey A, et al. Clinical and pharmacodynamic (PD) results of TEL0805 trial: a phase II study of telatinib (TEL) in combination with capecitabine $(X)$ and cisplatin $(\mathrm{P})$ as first-line treatment in patients (pts) with advanced gastric or gastroesophageal junction (GEJ) cancer. J Clin Oncol. 2011;29 Suppl:Abstr 4122.

46. Wilhelm SM, Carter C, Tang L, Wilkie D, McNabola A, Rong $\mathrm{H}$, et al. BAY 43-9006 exhibits broad spectrum oral antitumor activity and targets the RAF/MEK/ERK pathway and receptor tyrosine kinases involved in tumor progression and angiogenesis. Cancer Res. 2004;64:7099-109.

47. Qin S. Phase III study of apatinib in advanced gastric cancer: a randomized, double-blind, placebo-controlled trial. J Clin Oncol. 2014;32 Suppl:Abstr 4003.
48. Hu-Lowe DD, Zou HY, Grazzini ML, Hallin ME, Wickman GR, Amundson $\mathrm{K}$, et al. Nonclinical antiangiogenesis and antitumor activities of axitinib (AG-013736), an oral, potent, and selective inhibitor of vascular endothelial growth factor receptor tyrosine kinases 1, 2, 3. Clin Cancer Res. 2008;14: 7272-83.

49. Oh DY, Doi T, Shirao K, Lee KW, Park SR, Chen Y, et al. Phase I study of axitinib in combination with cisplatin and capecitabine in patients with previously untreated advanced gastric cancer. Cancer Res Treat. 2015;47:687-96.

50. Pick AM, Nystrom KK. Pazopanib for the treatment of metastatic renal cell carcinoma. Clin Ther. 2012;34:511-20.

51. Pavlakis N, Sjoquist KM, Tsobanis E, Martin A, Kang YK, Bang YJ, et al. INTEGRATE: a randomized phase II doubleblind placebo-controlled study of regorafenib in refractory advanced oesophagogastric cancer (AOGC): a study by the Australasian Gastrointestinal Trials Group (AGITG), first results. J Clin Oncol. 2015;33 Suppl 3:Abstr 9.

52. Wilhelm SM, Adnane L, Newell P, Villanueva A, Llovet JM, Lynch M. Preclinical overview of sorafenib, a multikinase inhibitor that targets both Raf and VEGF and PDGF receptor tyrosine kinase signaling. Mol Cancer Ther. 2008;7:3129-40.

53. Kim C, Lee JL, Choi YH, Kang BW, Ryu MH, Chang HM, et al. Phase I dose-finding study of sorafenib in combination with capecitabine and cisplatin as a first-line treatment in patients with advanced gastric cancer. Invest New Drugs. 2012;30: 306-15.

54. O'Farrell AM, Abrams TJ, Yuen HA, Ngai TJ, Louie SG, Yee KW, et al. SU11248 is a novel FLT3 tyrosine kinase inhibitor with potent activity in vitro and in vivo. Blood. 2003;101: 3597-605.

55. Morelli MP, Cascone T, Troiani T, Tuccillo C, Bianco R, Normanno $\mathrm{N}$, et al. Anti-tumor activity of the combination of cetuximab, an anti-EGFR blocking monoclonal antibody and ZD6474, an inhibitor of VEGFR and EGFR tyrosine kinases. J Cell Physiol. 2006;208:344-53.

56. Halmos B, Jia Y, Bokar JA, Fu P, Adelstein DJ, Juergens R, et al. A Phase I study of the combination of oxaliplatin/docetaxel and vandetanib for the treatment of advanced gastroesophageal cancer. Invest New Drugs. 2013;31:1244-50.

57. Al-Batran SE, Van Cutsem E, Oh SC, Bodoky G, Shimada Y, Hironaka S, et al. RAINBOW: a global, phase III, randomized, double-blind study of ramucirumab plus paclitaxel versus placebo plus paclitaxel patients with previously treated gastric or gastroesophageal junction (GEJ) adenocarcinoma: qualityof-life (QoL) results. J Clin Oncol. 2014;32 Suppl:Abstr 4058.

58. Bang YJ, Van Cutsem E, Feyereislova A, Chung HC, Shen L, Sawaki A, et al. Trastuzumab in combination with chemotherapy versus chemotherapy alone for treatment of HER2-positive advanced gastric or gastro-oesophageal junction cancer (ToGA): a phase 3, open-label, randomised controlled trial. Lancet. 2010;376:687-97.

59. Piccart-Gebhart MJ, Procter M, Leyland-Jones B, Goldhirsch A, Untch M, Smith I, et al. Trastuzumab after adjuvant chemotherapy in HER2-positive breast cancer. N Engl J Med. 2005;353:1659-72. 
60. Rohrberg KS, Pappot H, Lassen U, Westman M, Olesen RK, Pfeiffer $\mathrm{P}$, et al. Biomarkers in tissue from patients with upper gastrointestinal cancers treated with erlotinib and bevacizumab. Cancer Biol Ther. 2011;11:732-9.

61. Van Cutsem E, de Haas S, Kang YK, Ohtsu A, Tebbutt NC, Ming Xu J, et al. Bevacizumab in combination with chemotherapy as first-line therapy in advanced gastric cancer: a biomarker evaluation from the AVAGAST randomized phase III trial. J Clin Oncol. 2012;30:2119-27.

62. Bais C, Rabe C, Wild N, Swiatek-de Lange M, Chen D, Hong $\mathrm{K}$, et al. Comprehensive reassessment of plasma VEGFA (pVEGFA) as a candidate predictive biomarker for bevacizumab (Bv) in 13 pivotal trials (seven indications). J Clin Oncol. 2014;32 Suppl:Abstr 3040.

63. Hacker UT, Escalona-Espinosa L, Consalvo N, Goede V, Schiffmann L, Scherer SJ, et al. Evaluation of angiopoietin-2 as a biomarker in gastric cancer: results from the randomised phase III AVAGAST trial. Br J Cancer. 2016;114:855-62.

64. Fuchs CS, Tabernero J, Tomasek J, Chau I, Melichar B, Safran $\mathrm{H}$, et al. Candidate biomarker analyses in gastric or gastroesophageal junction carcinoma: REGARD trial of single-agent ramucirumab (RAM) vs. placebo (PL). J Clin Oncol. 2015;33 Suppl:Abstr 4029.

65. Jayson GC, Kerbel R, Ellis LM, Harris AL. Antiangiogenic therapy in oncology: current status and future directions. Lancet. 2016;388:518-29.

66. Ciliberto D, Staropoli N, Caglioti F, Gualtieri S, Fiorillo L, Chiellino S, et al. A systematic review and meta-analysis of randomized trials on the role of targeted therapy in the management of advanced gastric cancer: Evidence does not translate? Cancer Biol Ther. 2015;16:1148-59.

67. Chan D, Goldstein D, Sjoquist KM, Pavlakis N. Antiangiogenic agents (AAs) in metastatic oesophago-gastric cancer (mOGC): a systematic review and meta-analysis. J Clin Oncol. 2015;33 Suppl:Abstr e15111.

68. Hsu C, Shen YC, Cheng CC, Cheng AL, Hu FC, Yeh KH. Geographic difference in safety and efficacy of systemicchemotherapy for advanced gastric or gastroesophagealcarcinoma: a meta-analysis and meta-regression. Gastric Cancer. 2012;15:265-80.

69. Al-Refaie WB, Tseng JF, Gay G, Patel-Parekh L, Mansfield PF, Pisters PW, et al. The impact of ethnicity on the presentation and prognosis of patients with gastric adenocarcinoma. Results from the National Cancer Data Base. Cancer. 2008; 113:461-9.

70. Kim J, Sun CL, Mailey B, Prendergast C, Artinyan A, Bhatia S, et al. Race and ethnicity correlate with survival in patients with gastric adenocarcinoma. Ann Oncol. 2010;21:152-60.

71. Shah MA, Kelsen DP. Gastric cancer: a primer on the epidemiology and biology of the disease and an overview of the medical management of advanced disease. J Natl Compr Canc
Netw. 2010;8:437-47.

72. Gill S, Shah A, Le N, Cook EF, Yoshida EM. Asian ethnicityrelated differences in gastric cancer presentation and outcome among patients treated at a canadian cancer center. J Clin Oncol. 2003;21:2070-6.

73. Price TJ, Shapiro JD, Segelov E, Karapetis CS, Pavlakis N, Van Cutsem E, et al. Management of advanced gastric cancer. Expert Rev Gastroenterol Hepatol. 2012;6:199-208.

74. Kim R, Tan A, Choi M, El-Rayes BF. Geographic differences in approach to advanced gastric cancer: Is there a standard approach? Crit Rev Oncol Hematol. 2013;88:416-26.

75. Hironaka S, Ueda S, Yasui H, Nishina T, Tsuda M, Tsumura $\mathrm{T}$, et al. Randomized, open-label, phase III study comparing irinotecan with paclitaxel in patients with advanced gastric cancer without severe peritoneal metastasis after failure of prior combination chemotherapy using fluoropyrimidine plus platinum: WJOG 4007 trial. J Clin Oncol. 2013;31:4438-44.

76. Shitara K, Ikeda J, Yokota T, Takahari D, Ura T, Muro K, et al. Progression-free survival and time to progression as surrogate markers of overall survival in patients with advanced gastric cancer: analysis of 36 randomized trials. Invest New Drugs. 2012;30:1224-31.

77. Paoletti X, Oba K, Bang YJ, Bleiberg H, Boku N, Bouche O, et al. Progression-free survival as a surrogate for overall survival in advanced/ recurrent gastric cancer trials: a meta-analysis. J Natl Cancer Inst. 2013;105:1667-70.

78. Park SH. Second-line chemotherapy for patients with oesophagogastric adenocarcinoma. Lancet Oncol. 2014;15:8-10.

79. Kim SM, Park SH. Chemotherapy beyond second-line in advanced gastric cancer. World J Gastroenterol. 2015;21: 8811-6.

80. Shaked Y, Henke E, Roodhart JM, Mancuso P, Langenberg $\mathrm{MH}$, Colleoni M, et al. Rapid chemotherapy-induced acute endothelial progenitor cell mobilization: implications for antiangiogenic drugs as chemosensitizing agents. Cancer Cell. 2008;14:263-73.

81. Yasuda S, Sho M, Yamato I, Yoshiji H, Wakatsuki K, Nishiwada S, et al. Simultaneous blockade of programmed death 1 and vascular endothelial growth factor receptor 2 (VEGFR2) induces synergistic anti-tumour effect in vivo. Clin Exp Immunol. 2013;172:500-6.

82. Huang Y, Yuan J, Righi E, Kamoun WS, Ancukiewicz M, Nezivar J, et al. Vascular normalizing doses of antiangiogenic treatment reprogram the immunosuppressive tumor microenvironment and enhance immunotherapy. Proc Natl Acad Sci USA. 2012;109:17561-6.

83. Cristescu R, Lee J, Nebozhyn M, Kim KM, Ting JC, Wong SS, et al. Molecular analysis of gastric cancer identifies subtypes associated with distinct clinical outcomes. Nat Med. 2015;21: 449-56. 